Check for updates

Cite this: RSC Adv., 2017, 7, 18379
Received 15th January 2017 Accepted 18th March 2017

DOI: 10.1039/c7ra00610a

rsc.li/rsc-advances

\section{Loess surface grafted functional copolymer for removing basic fuchsin $\dagger$}

\begin{abstract}
Tingjun Lu, Li Wang, Yufeng He, ${ }^{*}$ Jing Chen and Rong-Min Wang (D)
Loess clay (LC), a very abundant clay with granules and high hydrophilicity, was modified by surface grafting copolymerization of functional monomers, such as acrylamide (AM) and sodium p-styrene sulfonate (StS), and a cross-linking agent ( $N, N$-methylenebisacrylamide: MBA), which afforded a LC surface grafting copolymer (LC-PAmS). Its structure and composition were characterized by Fourier transform infrared spectroscopy (FT-IR), thermal gravimetric analysis (TGA) and scanning electron microscopy (SEM). Acting as a novel and low-cost Loess-based polymer adsorbent, its adsorption behaviors were investigated with removing basic fuchsin (BF) in aqueous solution. After optimizing conditions, the removal rate got to $98.4 \%$ in $40 \mathrm{~min}$ at room temperature. Its adsorption mechanism was also investigated. It was found that the adsorption isotherm model could meet the Freundlich isotherm requirements and the dynamics were consistent with a pseudo-second-order kinetic model. In summary, LC-PAmS is a kind of polymer adsorbent for practically applied in wastewater treatment.
\end{abstract}

\section{Introduction}

As we know, dyes have made a great contribution to the progress of this beautiful and bright world as many commodities, such as dyestuffs, paper, textiles and plastics, have been industrially dyed. However, many kinds of dyes are classified as hazardous pollutants as they are very toxic even at very low concentrations. ${ }^{1}$ Basic fuchsin (BF), a water-soluble cationic dye, is widely used in textile industries for dyeing cotton, wool and silk. It is a great threat to aquatic organisms and also potentially harmful to humans as it can burn the eyes and irritate the skin. ${ }^{2}$ Therefore, it is necessary to remove BF from industrial wastewater before being discharged. Many techniques have been developed for the removal of dye, which include ion exchange, photocatalysis, chemical precipitation sonochemical degradation and adsorption..$^{3-5}$ Adsorption is a common and simple separation process for treatment of waste among them. ${ }^{6}$ The exploitation of novel, low costs and more effective adsorbents is always an important focus in this fields. ${ }^{7}$ Loess clay (LC), a typical silicate minerals, is a kind of rich soil on the earth. It shows a certain adsorptive property because of small granule and high hydrophilicity. In order to enhance adsorption capacity, the loess had been modified by surfactants. ${ }^{8-11}$ We found that LC could be modified by copolymer using in situ polymerization, which afforded a new kind of polymer/inorganic mineral composites. This Loessbased copolymer exhibited excellent adsorption activity for

Key Lab. Eco-Environment-Related Polymer Materials of Ministry of Education, College of Chemistry \& Chemical Engineering, Northwest Normal University, Lanzhou 730070 , China.E-mail: heyufeng@nwnu.edu.cn; wangrm@nwnu.edu.cn

$\uparrow$ Electronic supplementary information (ESI) available. See DOI: 10.1039/c7ra00610a removing $\mathrm{Pb}(\mathrm{II})$ ions. $^{12}$ However, in this polymer/inorganic mineral composites, the dosage of copolymer is relatively high, which increase the cost of polymer adsorbent. Here, in order to decrease the ratio of polymer, the surface grafting polymerization was employed to modify LC. Using acrylamide (AM) and sodium $p$-styrene sulfonate (StS) as functional monomers, $N, N$-methylenebisacrylamide (MBA) as cross-linking agent, the loess surface grafted copolymer (LC-PAmS) was successfully synthesized by grafting polymerization. Then, the obtained LC-PAmS was applied to remove $\mathrm{BF}$ from aqueous solutions, and optimal conditions, such as initial BF concentration and contact time on the LC-PAmS, were measured. Its adsorption mechanism was also investigated.

\section{Experimental section}

\subsection{Materials and reagents}

Loess clay (LC) was collected from the local hill near Lanzhou of China. It was ground and sifted through a 100-mesh sieve, and then stored in a desiccator for further use. 3-Methacryloxypropyl trimethoxysilane (KH-570), StS, AM and MBA were obtained commercially from Shanghai Zhongqin Chem. Reagent Co. Ltd, Shanghai Jingchun Reagent Co. Ltd, and Tianjin Baishi Chem. Ind. Co. Ltd, respectively. Potassium persulfate (KPS) was obtained from Yantai Shuangshang Chem. Ind. Co., Ltd BF, acetic acid (HAc), ammonia, hydrochloric acid ( $\mathrm{HCl}$ ) and ethanol are were commercially obtained with analytical grade.

\subsection{Preparation of LC-PAmS}

2.2.1 Acidizing of LC. The dried LC powder was treated by $\mathrm{HCl}$ aqueous solution $\left(4 \mathrm{~mol} \mathrm{~L}^{-1}\right)$ for $2 \mathrm{~h}$ at $80{ }^{\circ} \mathrm{C}$ with 
mechanical stirring. Then, LC was filtered and washed to neutral with distilled water. Being dried at $60^{\circ} \mathrm{C}$ for $5 \mathrm{~h}$, the $\mathrm{HCl}$ acidified loess (HLC) was obtained.

2.2.2 Surface grafting modification of LC. In $40 \mathrm{~mL}$ of EtOH/ $\mathrm{H}_{2} \mathrm{O}(3 / 1)$ solution, $5.0 \mathrm{~g}$ of HLC was dispersed for $0.5 \mathrm{~h}$ at room temperature. Its $\mathrm{pH}$ value was adjusted to 3-4 with HAc. Then, adding $1.0 \mathrm{~g}$ of $\mathrm{KH}-570$, the mixture was stirred for $30 \mathrm{~min}$. The $\mathrm{pH}$ value of the system was regulated to 9-10 by ammonia. The mixture was heated to $80{ }^{\circ} \mathrm{C}$ and kept for $3 \mathrm{~h}$ under continuous stirring. Then, the product was filtered and washed with ethanol for three times. After vacuum drying at $50{ }^{\circ} \mathrm{C}$, the silanized LC (LC-KH) was obtained and sealed in a tight plastic container for storage.

2.2.3 Surface grafting polymerization of loess. In protection of nitrogen, $18.0 \mathrm{~g}$ of LC-KH was dispersed in $50 \mathrm{~mL}$ of distilled water, and stirred $1 \mathrm{~h}$ at room temperature. Adding $1.0 \mathrm{~g}$ of StS, $2.0 \mathrm{~g}$ of AM and $0.16 \mathrm{~g}$ of MBA, the mixture was stirred for $0.5 \mathrm{~h}$, and gradually heated to $45{ }^{\circ} \mathrm{C}$ under nitrogen atmosphere. Then, adding $0.15 \mathrm{~g}$ of KPS, the reaction was continued by stirring $30 \mathrm{~min}$ at $75{ }^{\circ} \mathrm{C}$. The obtained loess surface grafting copolymer (LC-PAmS) was filtered and washed by $\mathrm{H}_{2} \mathrm{O}$ and $\mathrm{EtOH}$, respectively. Then the resultant product was dried to a constant weight at $60{ }^{\circ} \mathrm{C}$, milled and sifted through a 100-mesh sieve, and stored in a tight plastic container.

\subsection{Characterization of LC-PAmS}

FT-IR spectra of LC-PAmS were recorded between 400 and 4000 $\mathrm{cm}^{-1}$ through the $\mathrm{KBr}$ method with a FTS3000 spectrophotometer. Thermal gravimetric analysis (TGA) were carried out using TG analyzer (PerkinElmer, model Pyris Diamond, USA). A morphology analysis of certain features was visualized by using a SEM microscope (ULTRA Plus, at $5 \mathrm{kV}$, Germany).

\subsection{Adsorption behaviours}

Batch tests were conducted to investigate adsorption of LCPAmS for removing dye, and BF was selected as dye model. The content of residual BF was measured using a visible spectrum. The removal rate and adsorption capacity were calculated by eqn (1) and eqn (2).

$$
\begin{aligned}
& \text { Removal }(\%)=\frac{C_{0}-C_{\mathrm{e}}}{C_{0}} \times 100 \% \\
& \text { Adsorption capacity }=\frac{C_{0}-C_{\mathrm{e}}}{m} V
\end{aligned}
$$

where $C_{0}$ and $C_{\mathrm{e}}$ are the initial and equilibrium concentration of dyes ( $\mathrm{mg} \mathrm{L}^{-1}$ ) in the solution. $V$ is the volume of dyes solution (L) and $m$ is the weight of the adsorbent (g).

\section{Results and discussion}

\subsection{Preparation of LC surface grafting copolymer (LC-PAmS)}

As a kind of typical silicate minerals, loess is a sort of natural inorganic polymer adsorbent. It was found that the acid activation with $\mathrm{HCl}$ solution could dredge channel of loess, ${ }^{13}$ and improve its adsorption capacity. The acid activation could

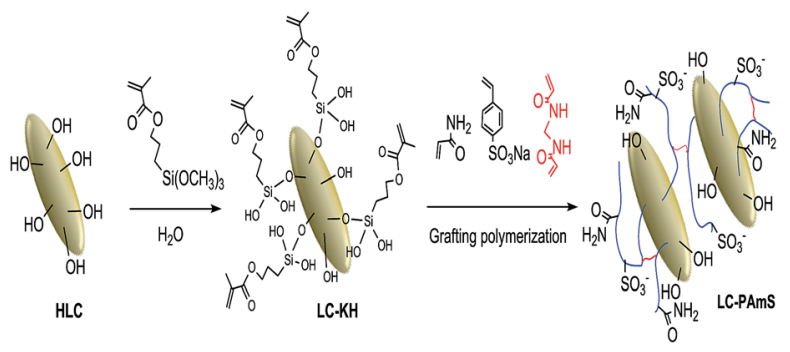

Scheme 1 Graft copolymerization on loess surface.

dissolve carbonate and other impurities in pores of loess, which increase the content of hydroxyl groups on clay surface. Therefore, we have also acidized LC before surface grafting modification. The reaction in preparation of LC-PAmS is showed in Scheme 1. Firstly, KH-570, a typical silane coupling agents with vinyl group, ${ }^{14}$ was grafted onto the LC surface, which allowed the double bond to be introduced. Secondly, using AM and StS as functional monomers, and MBA as cross-linking agent, the copolymer (PAmS) was grafted onto the LC surface by graft copolymerization, which afforded loess surface grafting copolymer (LC-PAmS). In copolymer chain of LC-PAmS, the highly hydrophilic group $\left(-\mathrm{SO}_{3}{ }^{-}\right)$of StS could improve its dispersity in aqueous environment and the salt resistance, and units of AM could improve its affinity to organic reagents. Using cross-linking agent, a few network structure could be formed between multiple linear copolymer, which is conducive to the adsorption.

In order to evaluate influence of different side groups in polymer chains for adsorption capacity, LC surface grafting polyacrylamide (LC-PAM) and LC surface grafting polystyrene sulfonate (PStS) were prepared by similar procedure (ESI $\dagger$ ).

\subsection{Characterization of LC-PAmS}

The FT-IR spectra of LC-PAmS and its materials (LC, HLC, LC$\mathrm{KH})$ were measured and showed in Fig. S1.† For HLC, the absorption peaks at $3645 \mathrm{~cm}^{-1}, 3452 \mathrm{~cm}^{-1}$ and $1631 \mathrm{~cm}^{-1}$ are correspond to the $-\mathrm{OH}$ stretching vibrations and bending vibrations. A sharp peak at $797 \mathrm{~cm}^{-1}$ can be assigned to quartz. The bands observed at $1093 \mathrm{~cm}^{-1}, 538 \mathrm{~cm}^{-1}$ and $479 \mathrm{~cm}^{-1}$ can be assigned to the $\mathrm{Si}-\mathrm{O}-\mathrm{Si}$ stretching vibrations and bending vibrations. It was found that main characteristic peaks in LC$\mathrm{KH}$ and LC-PAmS are similar to HLC as silicate structure was retained basically. In LC-KH, there are five peaks from 3000 $\mathrm{cm}^{-1}$ to $2800 \mathrm{~cm}^{-1}$, which are attributed to $=\mathrm{C}-\mathrm{H}\left(\nu_{\mathrm{s}}\right),-\mathrm{CH}_{3}\left(\nu_{\mathrm{s}}\right)$, $-\mathrm{CH}_{3}\left(\nu_{\mathrm{as}}\right),-\mathrm{CH}_{2}\left(\nu_{\mathrm{s}}\right)$, and $-\mathrm{CH}_{3}\left(\nu_{\mathrm{as}}\right)$, of $\mathrm{KH}-570$, respectively. The characteristic peaks near, $1720,1600 \mathrm{~cm}^{-1}$ are attributed to ester carbonyl and $\mathrm{C}=\mathrm{C}$ stretching vibrations, respectively. In LC-PAmS, the peak at $1183 \mathrm{~cm}^{-1}$ represents sulfonate group $\left(-\mathrm{SO}_{3}{ }^{-}\right)$of StS in polymer chain. The bands near $1670 \mathrm{~cm}^{-1}$ and $1450 \mathrm{~cm}^{-1}$ are attributed to amide $(\mathrm{C}=\mathrm{O}$ stretching $)$ and $\mathrm{C}-\mathrm{N}$ stretching vibrations conferred by AM. The characteristic peak of $=\mathrm{C}-\mathrm{H}$ stretching vibrations near $3000 \mathrm{~cm}^{-1}$ disappears in LC-PAmS. It indicates that $\mathrm{C}=\mathrm{C}$ groups of $\mathrm{LC}-\mathrm{KH}$ had copolymerized with Am and StS. And it means copolymers of AM and StS have been successfully grafted onto the loess surface. 

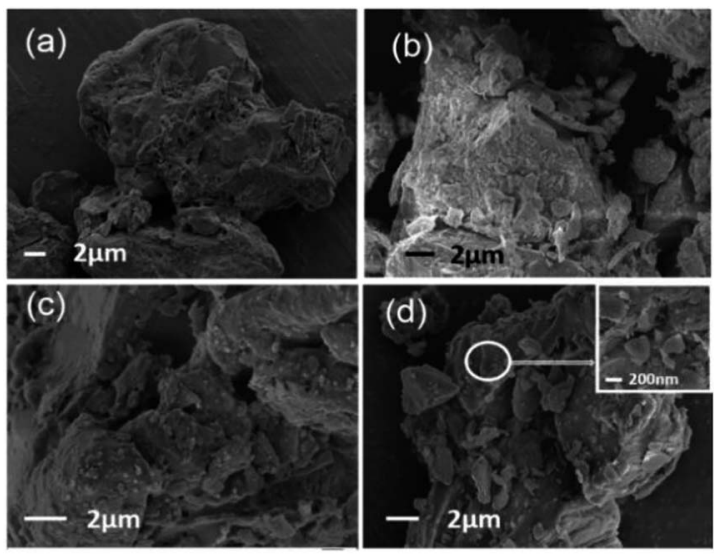

Fig. 1 SEM images of LC (a), HLC (b), LC-KH (c) and LC-PAmS (d).

TG and DTG curves of LC-PAmS and its materials (LC, HLC, LC-KH) are showed in Fig. S2. $\dagger$ It is obviously that LC-PAmS is not stable than its materials. There are only a few weight loss in LC and HLC.

The weight loss of LC-KH increase to $3.2 \%$, which indicates KH-570 was grafted onto LC particles. The total weight loss of LC-PAmS till $800{ }^{\circ} \mathrm{C}$ is only $13.1 \%$, and most of them losing from $300{ }^{\circ} \mathrm{C}$ to $500{ }^{\circ} \mathrm{C}$ is degradation of polymer chains. Compared with polymer/loess composites, ${ }^{12}$ the content of polymer in loess surface grafting copolymer (LC-PAmS) decreased greatly.

SEM images of LC, HLC, LC-KH and LC-PAmS (d) are showed in Fig. 1. Obviously, the surface of LC (a) is rough. Particles of HLC (b) show clear boundary, and there are a lot of tiny crack. The morphology of LC-KH (c) is not much different to HLC because LC was modified by small molecular $\mathrm{KH}-570$ on surface. However, the loess surface of LC-PAmS is wrapped by a layer of polymer film. This suggests that the copolymer was successfully grafted onto the surface of loess.

\subsection{Adsorption behaviours of LC-PAmS}

LC-PAmS was applied to remove BF, a typical dye, in aqueous solution. In order to investigate the influence of functional groups in polymer chains, the adsorption behaviours of LCPStS, LC-PAM, and its materials were also measured. The results are showed in Table 1.

The adsorption capacity of LC-PAmS is higher than that of materials (LC, HLC). It is also higher than that LC surface

Table 1 The absorption ability of LC-PAmS and its materials ${ }^{a}$

\begin{tabular}{lll}
\hline Adsorbents & $\begin{array}{l}\text { Removal } \\
(\%)\end{array}$ & $\begin{array}{l}\text { Adsorption capacity } \\
\left(\mathrm{mg} \mathrm{g}^{-1}\right)\end{array}$ \\
\hline LC & 27.60 & 13.77 \\
HLC & 44.36 & 22.18 \\
LC-PAM & 46.70 & 23.35 \\
LC-PStS & 41.80 & 20.90 \\
LC-PAmS & 94.98 & 47.49
\end{tabular}

${ }^{a}$ Adsorbents: $0.05 \mathrm{~g} / 50 \mathrm{~mL}$; time: $30 \mathrm{~min}$; temp: $25^{\circ} \mathrm{C}$.

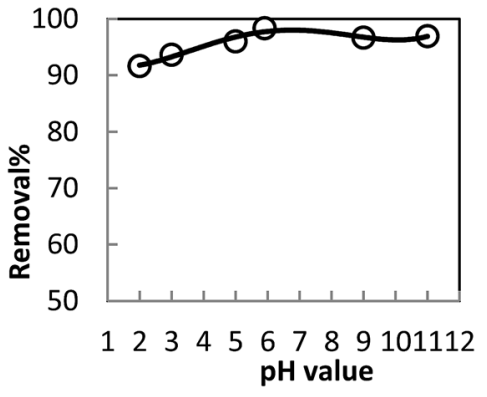

Fig. 2 Effect of $\mathrm{pH}$ value on adsorption ([BF] $]_{\text {Init }}: 100 \mathrm{mg} \mathrm{L}^{-1}$, adsorbent: $0.07 \mathrm{~g} / 50 \mathrm{~mL}$, temp: $25^{\circ} \mathrm{C}$, time: $40 \mathrm{~min}$ ).

grafting polyacrylamide (LC-PAM) and LC surface grafting polystyrene sulfonate (LC-PStS). We consider there are synergistic effects among two kinds of functional groups when LCPAmS removes dyes.

In order to optimize conditions for removing $\mathrm{BF}$, some parameters for affecting the adsorption process, such as initial concentration dyes, adsorbent dosage, contact time, temperatures, $\mathrm{pH}$ value were investigated.

3.3.1 Effect of $\mathbf{p H}$ value. The $\mathrm{pH}$ value of the solution is an important factor, which can determine the surface charge of the adsorbent. ${ }^{15}$ The effect of $\mathrm{pH}$ value on adsorption of BF by LC-PAmS was measured from 2.00 to 11.00 . And results are presented in Fig. 2. It shows the removal rate increased with increasing pH from 2.00 to 5.91 (natural pH of BF solution). The low removal rate at acidic solution is attributed to the competition of hydrogen ion with cationic dye $(\mathrm{BF}){ }^{\mathbf{1 6}}$ In alkaline solution, the removal rate tend to be unchanging. Therefore, the natural BF solution was used in following measurement.

3.3.2 Effect of adsorbent dosage. Influence of adsorbent dosage on removal rate of $\mathrm{BF}$ is showed in Fig. 3. With increasing the sorbent dosage from 0.01 to $0.07 \mathrm{~g} / 50 \mathrm{~mL}$, the removal rate of BF increased from 34.03 to $98.46 \%$. It is due to the increase of active sites on the adsorbent. ${ }^{17}$ The adsorption rate became constant when the dosage was more than $0.07 \mathrm{~g} / 50 \mathrm{~mL}$.

3.3.3 Effect of initial concentration. Fig. 4 shows the adsorption capacity of LC-PAmS with varying initial concentration of BF. With increasing $[\mathrm{BF}]_{\text {Init }}$ from 20 to $200 \mathrm{mg} \mathrm{L}^{-1}$, the

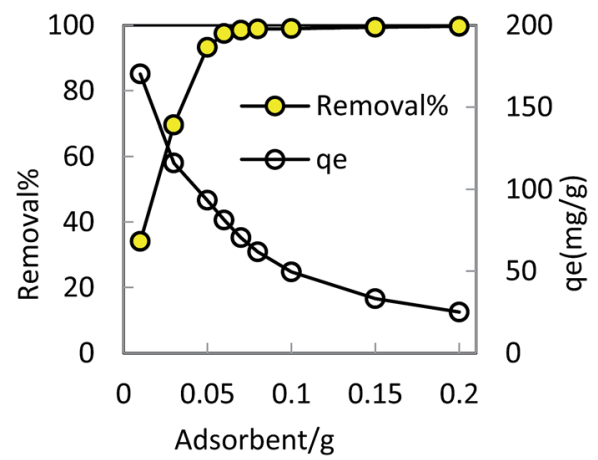

Fig. 3 Effect of adsorbent dosage on adsorption of BF ([BF] $]_{\text {|nit }}: 100 \mathrm{mg}$ $\mathrm{L}^{-1}$, temp: $25^{\circ} \mathrm{C}$, time: $\left.40 \mathrm{~min}\right)$. 


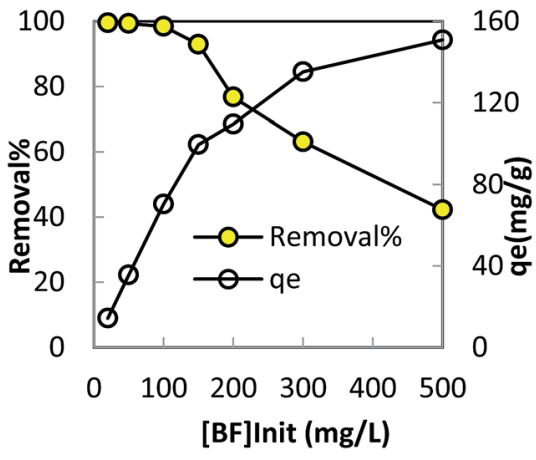

Fig. 4 Effect of initial concentrations of BF. (Adsorbent: $0.07 \mathrm{~g} / 50 \mathrm{~mL}$, temp: $25^{\circ} \mathrm{C}$, time: $\left.40 \mathrm{~min}\right)$.

adsorption capacity increased from 14.22 to $109.58 \mathrm{mg} \mathrm{g}^{-1}$. This can be attributed to mass transfer effects and the driving force of the concentration gradient being directly proportional to the initial concentrations. ${ }^{18}$ The optimum $[\mathrm{BF}]_{\text {Init }}$ was $100 \mathrm{mg} \mathrm{L}^{-1}$.

3.3.4 Effect of contact time. Contact time is an important parameter because fast adsorption rate is also indispensable for practical application. ${ }^{\mathbf{1 9 2 0}}$ The effect of contact time on BF removal efficiency was investigated and the results are showed in Fig. 5. It shows the removal rate increased rapidly within $40 \mathrm{~min}$. The initial rapid phase is probably due to the abundant availability of reaction sites. After that, an equilibrium condition was gradually approached in a slow process. As the surface adsorption sites became exhausted, the rate of uptake was

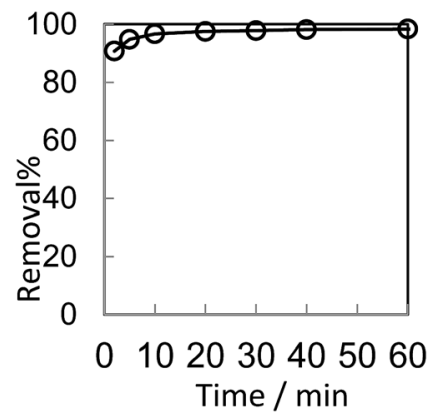

Fig. 5 The influence of time on adsorption of $B F\left([B F]_{\text {|nit }}: 100 \mathrm{mg} \mathrm{L}^{-1}\right.$, adsorbent: $0.07 \mathrm{~g} / 50 \mathrm{~mL}$, temp: $25^{\circ} \mathrm{C}$ ).

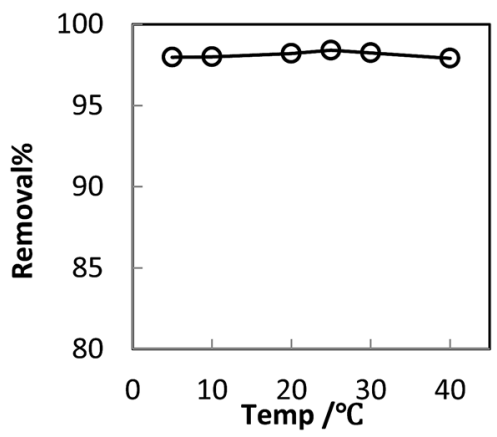

Fig. 6 The influence of temperature on adsorption of $B F\left([B F]_{\text {Init }}\right.$ : $100 \mathrm{mg} \mathrm{L}^{-1}$, adsorbent: $0.07 \mathrm{~g} / 50 \mathrm{~mL}$, time: $40 \mathrm{~min}$ ). controlled by that of transport from the exterior sites to the interior of the adsorbent particles.

3.3.5 Effect of temperature. Effect of temperature on adsorption is showed in Fig. 6. It is found that the removal rate change lightly in the range of $97.9-98.4 \%$ with increasing temperature from 5 to $40{ }^{\circ} \mathrm{C}$. That means the temperature have little effect on LC-PAmS absorbing dyes. Therefore, the LC-PAmS has a wide temperature scope for adsorption of BF.

\subsection{Adsorption isotherm study}

Adsorption isotherms are important for describing adsorption process of adsorbate on adsorbent surface. Hence, the correlation of equilibrium data using either a theoretical or empirical equation is essential for the adsorption interpretation and prediction of the extent of adsorption. ${ }^{21}$ The process for removing BF by LC-PAmS was evaluated with nonlinear form of Langmuir (eqn (3)) and Freundlich (eqn (4)) isotherm models:

Langmuir:

$$
q_{\mathrm{e}}=\frac{q_{\mathrm{m}} K_{\mathrm{L}} C_{\mathrm{e}}}{1+K_{\mathrm{L}} C_{\mathrm{e}}}
$$

Freundlich:

$$
q_{\mathrm{e}}=K_{\mathrm{f}} C_{\mathrm{e}}^{1 / n}
$$

where $q_{\mathrm{e}}$ is the adsorbed amount of BF at equilibrium $\left(\mathrm{mg} \mathrm{g}^{-1}\right)$, $q_{\mathrm{m}}$ is the maximum adsorption capacity $\left(\mathrm{mg} \mathrm{g}^{-1}\right), C_{\mathrm{e}}$ is the equilibrium BF concentration in solution $\left(\mathrm{mg} \mathrm{L}^{-1}\right)$, and $K_{\mathrm{L}}$ is the Langmuir isotherm constant $\left(\mathrm{L} \mathrm{mg}^{-1}\right)$, which is related to the adsorption equilibrium. $K_{\mathrm{f}}\left(\mathrm{L} \mathrm{g}^{-1}\right)$ and $n$ are Freundlich constants related to the capacity and intensity of adsorption, respectively.

The plots of Langmuir and Freundlich for adsorption of BF are showed in Fig. 7. The correlation coefficients of Langmuir and Freundlich are 0.8769 and 0.9540 , respectively, which indicate the best fitting of the data to the Freundlich equation. This phenomenon suggests that heterogeneous adsorption take place on the surface of LC-PAmS.

\subsection{Adsorption kinetics}

Adsorption kinetics is one of characteristics for defining adsorption efficiency and explaining adsorption mechanism. Here, the nonlinear forms of pseudo-first-order and pseudo-
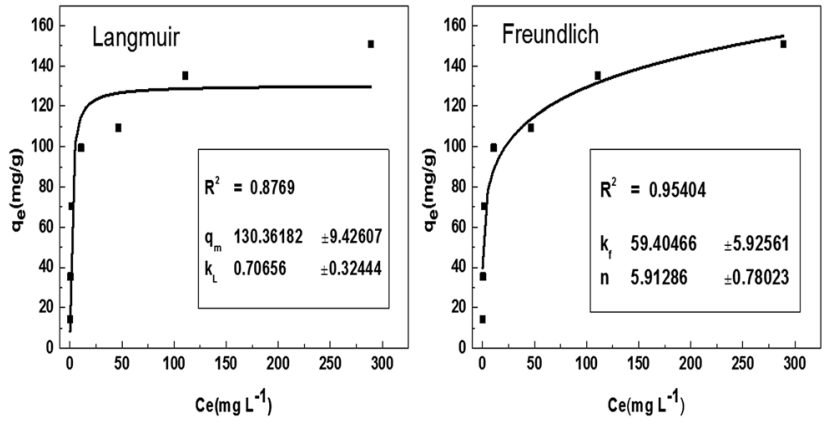

Fig. 7 Isotherms models for BF adsorption. 

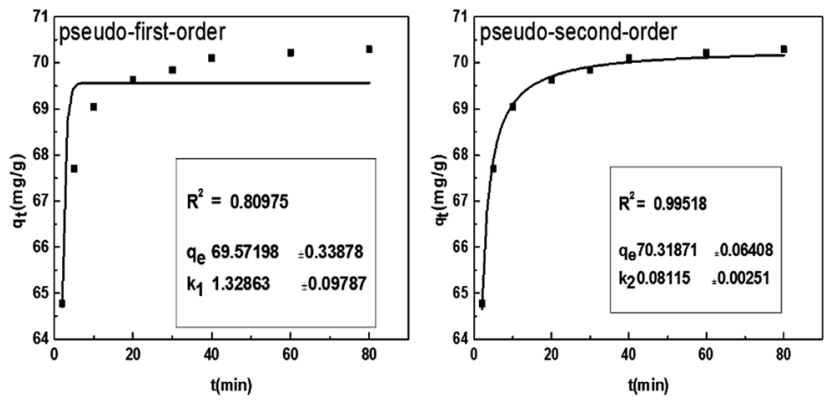

Fig. 8 Kinetic models for BF adsorption.

second-order kinetics were used to test the experimental data. The pseudo-first-order model can be explained as follows:

$$
q_{\mathrm{t}}=q_{\mathrm{e}}-\exp ^{k_{1} t}
$$

where $q_{\mathrm{e}}$ and $q_{\mathrm{t}}$ are the amounts of BF adsorbed $\left(\mathrm{mg} \mathrm{g}^{-1}\right)$ at equilibrium and time $t(\mathrm{~min})$, respectively. $k_{1}$ is the adsorption rate constant for the first-order adsorption.

The pseudo-second-order model can be expressed as follows:

$$
q_{t}=\frac{q_{\mathrm{e}}^{2} k_{2} t}{1+q_{\mathrm{e}} k_{2} t}
$$

where $k_{2}$ is the second order reaction constant.

The analysis of the data are showed in Fig. 8 by plotting $q_{\mathrm{t}}$ as a function of the contact time $t$. The value of correlation coefficient $\left(R^{2}\right)$ obtained from pseudo-second-order kinetics is higher than that of pseudo-first-order kinetics and the calculated $q_{\mathrm{e}, \mathrm{cal}}\left(70.32 \mathrm{mg} \mathrm{\textrm {g } ^ { - 1 }}\right)$ is closer to experimental $q_{\text {e,exp }}\left(70.30 \mathrm{mg} \mathrm{g}^{-1}\right)$. It indicates that the pseudo-second-order model is appropriate for the adsorption phenomena.

\section{Conclusions}

The copolymer of AM and StS was grafted onto surface of loess particles by graft copolymerization. It was found that the obtained loess surface grafted functional copolymer (LC-PAmS), was an effective adsorbent for the removal of basic fuchsin from aqueous solutions. The removal rate reached to $98.4 \%$. Kinetic and isotherm studies were carried out. The adsorption isotherms of LC-PAmS for basic fuchsin could be described well by the Freundlich model. The experimental data fitted the pseudo-second-order kinetic model very well. In summary, LCPAmS is a kind of polymer adsorbent for removing BF from solution and will be practically applied in wastewater treatment.

\section{Acknowledgements}

The project was supported by the National Natural Science Foundation $(21364012 ; 21263024)$ of China.

\section{References}

1 (a) G. P. Hao, W. C. Li, S. Wang, S. Zhang and A. H. Lu, Carbon, 2010, 48, 3330; (b) Y. F. Hao, L. G. Yan, H. Q. Yu,
K. Yang, S. J. Yu, R. R. Shan and B. Du, J. Mol. Liq., 2014, 199, 202; (c) V. Chandane and V. K. Singh, Desalin. Water Treat., 2016, 57, 4122.

2 (a) T. A. Khan and E. A. Khan, Appl. Clay Sci., 2015, 107, 70; (b) Y. He, H. Li, Z. Zhang, T. Lu and R. M. Wang, Key Eng. Mater., 2017, 726, 345.

3 (a) S. Raghu and C. A. Basha, J. Hazard. Mater., 2007, 149, 324; (b) Y. Dong, Z. Han, C. Liu and F. Du, Sci. Total Environ., 2010, 408, 2245; (c) M. X. Zhu, L. Lee, H. H. Wang and Z. Wang, J. Hazard. Mater., 2007, 149, 735.

4 A. Taamallah, S. Merouani and O. Hamdaoui, Desalin. Water Treat., 2016, 57, 27314.

5 L. H. Huang, J. Kong, W. L. Wang, C. L. Zhang, S. Niu and B. Y. Gao, Desalination, 2012, 286, 268.

6 (a) A. S. K. Kumar, R. Ramachandran, S. Kalidhasan, V. Rajesh and N. Rajesh, Chem. Eng. J., 2012, 211, 396; (b) Y. F. He, F. Li, R. M. Wang, F. Li, Y. Wang and Z. Zhang, Water Sci. Technol., 2010, 61, 1235.

7 X. Yang, Y. H. Li, Q. J. Du, J. K. Sun, L. Chen, S. Hu, Z. H. Wang and L. H. Xia, J. Colloid Interface Sci., 2015, 453, 107.

8 H. Chen, R. Yang, K. Zhu, W. Zhou and M. Jiang, J. Hazard. Mater., 2002, 94, 91.

9 Q. Yang, J. Zhang, Q. Yang, Y. Yu and G. Yang, Desalin. Water Treat., 2012, 39, 10.

10 J. H. Park and D. I. Jung, Desalination, 2011, 269, 104.

11 T. C. An, H. Chen, H. Y. Zhan and R. Berndtsson, Environ. Geol., 2005, 47, 467.

12 Y. F. He, L. Zhang, R. M. Wang, H. R. Li and Y. Wang, J. Hazard. Mater., 2012, 227, 334.

13 H. M. Yang, A. D. Tang, J. O. Ouyang, M. Li and S. Mann, J. Phys. Chem. B, 2010, 114, 2390.

14 (a) S. Kango, S. Kalia, A. Celli, J. Njuguna, Y. Habibi and R. Kumar, Prog. Polym. Sci., 2013, 38, 1232; (b) X. Jin, C. Yu, Y. Li, Y. Qi and L. Yang, J. Hazard. Mater., 2011, 186, 1672; (c) Y. Zhao, Y. Chen, M. Li, S. Zhou, A. Xue and W. Xing, J. Hazard. Mater., 2009, 171, 640.

15 Ş. Tokalığlu, E. Yavuz, A. Aslantaş, H. Şahan, F. Taşkin and Ş. Patat, Spectrochim. Acta, Part A, 2015, 149, 378.

16 (a) Y. B. Song, X. D. Song, C. J. Cheng and Z. G. Zhao, RSC $A d v$., 2015, 5, 87030; (b) H. Qiao, Y. M. Zhou, F. Yu, E. A. Wang, Y. H. Min, Q. Huang, L. F. Pang and T. S. Ma, Chemosphere, 2015, 141, 297.

17 (a) J. Xiao, H. Zhang, Y. Xia, Z. Li and W. Huang, RSC Adv., 2016, 6, 39861; (b) M. El Haddad, J Taibah Univ. Sci., 2016, 10, 664 .

18 L. H. Huang, J. J. Kong, W. L. Wang, C. L. Zhang, S. F. Niu and B. Y. Gao, Desalination, 2012, 286, 268.

19 Y. Liu, Y. Kang, B. Mu and A. Q. Wang, Chem. Eng. J., 2014, 237, 403.

20 W. J. He, Y. F. He, D. Z. Yan, Y. Wang and R. M. Wang, J. Dispersion Sci. Technol., 2014, 35, 1378.

21 (a) L. Wang, W. J. He, Y. F. He, H. Li and R. M. Wang, Key Eng. Mater., 2015, 633, 165; (b) Y. Bulut and H. Karaer, J. Dispersion Sci. Technol., 2015, 36, 61. 